\title{
Percepção social em EAD: Identificando necessidades para o LMS Amadeus
}

Title: Social Perception in Distance Learning: Identifying Needs for Amadeus LMS

\author{
Ivanildo José de Melo Filho \\ Instituto Federal de Pernambuco - Campus Belo Jardim \\ UFPE - Centro de Informática \\ ivanildo.melo@belojardim.ifpe.edu.br
}

\author{
Alex Sandro Gomes \\ UFPE - Centro de Informática \\ asg@cin.ufpe.br
}

\author{
Rosângela Saraiva Carvalho \\ UFPE - Centro de Informática \\ Escola Superior de Relações Públicas de Pernambuco \\ rosangelac@gmail.com
}

\author{
Rosangela Maria de Melo \\ Instituto Federal de Pernambuco - Campus Belo Jardim \\ ITEP - Instituto Tecnológico de Pernambuco \\ rmdemelo@gmail.com
}

\begin{abstract}
Resumo O presente trabalho tem como objetivo avaliar os mecanismos de percepção social existentes no Ambiente Virtual de Aprendizagem Amadeus, e averiguar a contribuição destes para comunicação e o engajamento dos alunos no ambiente. Os participantes desta pesquisa são alunos do último ano do curso técnico do IFPE - Campus Belo Jardim. O procedimento metodológico aplicado neste trabalho consistiu na interação com o ambiente, aplicação de questionário, realização de grupo focal e observação. A partir das necessidades identificadas, foi possivel estabelecer novos requisitos e complementar funcionalidades de percepção social existentes no ambiente. Todos os resultados foram prototipados para o LMS Amadeus.
\end{abstract}

Palavras-Chave: percepção social, identificação de necessidades, prototipação de requisitos, LMS Amadeus

\begin{abstract}
This paper is intended to evaluate the social awareness mechanisms existing on the virtual learning environment Amadeus as well as to verify the contribution of such mechanisms to the communication and engagement of students on current the environment. This research involves the IFPE last year students of technical course - Belo Jardim Campus. The methodological approach used on this work consisted in the interaction with the environment, questionnaires, focus groups and observation. From the identified needs, it was possible to establish new requirements and additional features of social perception in the environment. All results were prototyped for Amadeus LMS.
\end{abstract}

Keywords: social awareness, identifying needs, prototyping of requirements, LMS Amadeus 


\section{Introdução}

Com a crescente sofisticação dos Learning Management Systems (LMS) ou Ambientes Virtuais de Aprendizagem (AVA), a educação a distância e a presencial tem à sua disposição recursos que proporcionam o ensino e a aprendizagem com mais eficácia aos participantes, tendo em vista a utilização e familiarização de ferramentas tecnológicas que permitem explorar oportunidades para a colaboração e engajamento dos participantes de diversas formas. Conforme [1], estes sistemas transformaram as relações de tempo e espaço e a relação entre aluno e docente que, nesse contexto, pode extrapolar a sala de aula.

De acordo com [2], desde meados da década de 1980, a conscientização e sensibilização para os sistemas de colaboração tem sido um ponto focal para as pesquisas na área de Interação Humano-Computador (IHC). De acordo com os autores, os primeiros anos de pesquisa focaram principalmente na descoberta de como a percepção é importante para a colaboração, principalmente através de estudos de campo e pela crescente utilização das tecnologias de comunicação em rede.

Segundo [3], os elementos de percepção são os elementos do espaço compartilhado através dos quais são disponibilizadas as informações destinadas a prover percepção. Os autores enfatizam que o ambiente de aprendizagem colaborativa deve fornecer elementos de percepção que disponibilizem de maneira adequada as informações necessárias à colaboração e ao trabalho individual. Dessa maneira, norteado pela sua percepção, os participantes criam um entendimento compartilhado e se coordenam de forma que seus esforços individuais acrescentem valor ao trabalho coletivo.

Para [4], a percepção social abrange a consciência da situação social de um grupo ou comunidade em um ambiente compartilhado, que pode ser física, virtual ou ambas. Permite ainda, o mapeamento das funções, atividades, status e as relações sociais dos seus participantes. É sabido que a percepção social objetiva ajudar as pessoas a construírem e manterem a consciência das atividades umas das outras, no contexto ou situação, ainda que estes não estejam presentes no mesmo ambiente [3];[4];[5];[6] e [7].

Neste sentido, os elementos de percepção social contidos nos ambientes virtuais de aprendizagem (AVA) podem ser definidos como mecanismos destinados a auxiliar as pessoas construírem e manterem a consciência das atividades uns dos outros, o contexto ou situação, mesmo quando os participantes não estão no ambiente de aprendizagem [7]. Além disso, segundo [8], os elementos de percepção social proporcionam benefícios, tais como: o aumento da eficácia da colaboração entre os participan- tes, promovendo as relações sociais e melhorado o bemestar dos indivíduos, no sentido de eliminar a sensação de estar sozinho no ambiente de aprendizagem.

É importante ressaltar que as atividades de colaboração podem ser ampliadas nos ambientes virtuais de aprendizagem, desde que seus elementos de percepção social estejam dispostos e permitam os participantes compartilharem saberes e experiências, originando assim, o aprendizado coletivo, motivando a prática das suas habilidades e competências, além de permitir a reelaboração e construção conjunta de novos conhecimentos.

Sendo assim, o presente trabalho tem como objetivo avaliar os elementos de percepção social existente no ambiente virtual de aprendizagem LMS Amadeus, de modo a identificar necessidades que podem ser complementadas ou sugeridas aos elementos de percepção existentes no ambiente.

Esta pesquisa está organizada da seguinte forma: a seção 2 apresenta o LMS Amadeus, a seção 3 trata do método adotada nesta pesquisa; a seção 4 apresenta os resultados, a seção 5 , os requisitos estabelecidos a partir das necessidades. A seção 6 traz sugestões de protótipos baseadas nos requisitos estabelecidos, e, por fim,a seção 7 evidencia as considerações finais.

\section{Ambiente Virtual de Aprendizagem - LMS Amadeus}

O ambiente virtual de aprendizagem Amadeus tem origem em um conjunto de pesquisas acadêmicas na área de Interação Humano Computador e Tecnologia Educacional, desenvolvidas pelo grupo de pesquisa $\mathrm{CCTE}^{1}$ Ciências Cognitivas e Tecnologia Educacional do Centro de Informática da UFPE - Universidade Federal de Pernambuco.

Esse ambiente virtual de aprendizagem é definido por [9] como um sistema de gestão de aprendizagem para educação presencial, a distância e todas as suas variações. Ele é baseado no conceito de blended learning, segundo o qual, para se atingir um grande público com projetos de formação à distância, faz-se necessário uma combinação de formas de mediar à apresentação e interações com os conteúdos das aulas.

A interação entre os usuários, e destes com o conteúdo no ambiente permite a execução de novas estratégias de ensino e de aprendizagem orientadas por teorias construtivistas ou sócio-interacionista do desenvolvimento humano.

Ainda em [9] são descritas as principais características

\footnotetext{
1 CCTE na Internet: <http:/www.cin.ufpe.br/ ccte>
} 
do LMS Amadeus:

- Interface simplificada e intuitiva, concebida com usabilidade e desenvolvida com tecnologias da Web 2.0 e $\mathrm{AJAX}^{1}$;

- Uso de uma ampla gama de mídias, desde os tradicionais chats até a discussão simultânea entre vários usuários que estão assistindo ao mesmo vídeo, por exemplo;

- Compartilhamento de vídeos em situações de colaboração síncrona;

- Servidor de jogos multiusuários promovendo formas alternativas de interação com mídias de jogos;

- Sistema de controle de experimentos e medida em tempo real pela internet;

- Percepção da atividade social na interface web e nos diversos ambientes interligados;

- Mobile learning: estilos de interação por meio de dispositivos móveis;

Atualmente o LMS Amadeus é distribuído sob uma licença de Software Livre e desde março de 2009, passou a integrar o PSPB2 - Portal do Software Público Brasileiro. A comunidade do Projeto Amadeus no PSPB conta com mais de 5000 membros e com colaborações internacionais de países como: Irlanda, Chile, Alemanha e França.

\section{Método}

A metodologia aplicada neste trabalho buscou avaliar a natureza dos elementos de percepção social e de colaboração existentes no LMS Amadeus por meio da perspectiva dos participantes.

Com caráter exploratório e utilizando uma abordagem qualitativa, esta pesquisa é classificada por [10] e [11] como pesquisa não-experimental. O desenho metodológico que foi aplicado nesta pesquisa, durante o acesso ao campo foi planejado de forma que, ao longo do seu desenvolvimento pudessem ocorrer interações com o universo participante. Para tanto, foram utilizadas técnicas de observação, questionários, grupo focal, gravação das ações em tela do computador.

\subsection{Contexto}

O contexto deste trabalho esteve centrado na identifi-

1 AJAX - é o uso metodológico de tecnologias, providas por navegadores, para tornar páginas web mais interativas com o usuário.

${ }^{2}$ Portal do Software Público Brasileiro na Internet: http://www.softwarepublico.gov.br. cação dos fenômenos de percepção social no processo de navegação das interfaces web do LMS Amadeus.

No ambiente, os participantes da pesquisa navegaram por uma sequência de telas - vide Figura 1 - que dispõe de informações acerca do curso, denominado "[IFPEBelo Jardim] Capacitação Amadeus", desde a entrada no ambiente até a busca de informações sobre o curso a ser realizado.

A sequência de telas apresentada pela Figura 1 é formada pelas interfaces: $\log \mathrm{in}^{3}$ no LMS Amadeus, seleção do curso, solicitação de matrícula no curso, visualização dos colegas de sala e pelas informações do curso ou módulo.

A pesquisa foi realizada no Instituto Federal de Pernambuco - IFPE Campus Belo Jardim e contou com a participação de 9 alunos do último ano do curso técnico em informática utilizando a disciplina de Eletricidade e Eletrônica Básica. O Quadro 1 descreve as características dos participantes envolvidos na pesquisa.

\begin{tabular}{|c|c|c|c|}
\hline IDADE & $\begin{array}{l}\text { MODALIDADE } \\
\text { DE ENSINO }\end{array}$ & $\begin{array}{c}\text { EXPERIÊNCIA } \\
\text { COM LMS }\end{array}$ & $\begin{array}{c}\text { EXPERIÊNCIA } \\
\text { COM FERRAMEN- } \\
\text { TAS COLABORA- } \\
\text { TIVAS }\end{array}$ \\
\hline 16 & Concomitante ${ }^{4}$ & Já utilizou & Sim \\
\hline 16 & Concomitante & Já utilizou & Sim \\
\hline 16 & Concomitante & Já utilizou & Sim \\
\hline 16 & Concomitante & $\begin{array}{c}\text { Conhece por } \\
\text { outras fon- } \\
\text { tes }\end{array}$ & Sim \\
\hline 17 & Concomitante & $\begin{array}{l}\text { Conhece por } \\
\text { outras fon- } \\
\text { tes }\end{array}$ & Sim \\
\hline 17 & Concomitante & $\begin{array}{c}\text { Conhece por } \\
\text { outras fon- } \\
\text { tes }\end{array}$ & Sim \\
\hline 19 & Concomitante & $\begin{array}{l}\text { Conhece por } \\
\text { outras fon- } \\
\text { tes }\end{array}$ & Sim \\
\hline 19 & Concomitante & Já utilizou & Sim \\
\hline 19 & Subseqüente ${ }^{6}$ & Já utilizou & Sim \\
\hline
\end{tabular}

Quadro 1 - Características dos participantes.

Foram definidas 3 (três) sessões de interação no LMS Amadeus com duração de 60 minutos em dias distintos. Nesta fase, os participantes utilizaram um laboratório do

\footnotetext{
${ }^{3}$ Login - Entrada no sistema.

${ }^{4}$ Modalidade de ensino concomitante - modalidade de cursos destinados a alunos que tenham concluído o ensino fundamental.

5 Outras fontes - (amigos, internet, outros meios).

${ }^{6}$ Modalidade de ensino subsequente - modalidade de cursos destinados a que tenham concluído o ensino médio.
} 
IFPE - Campus Belo Jardim, todos em computadores individuais, quando então, foram simuladas atividades a distância. Em cada encontro, cada participante recebeu um documento de orientação que descrevia as instruções sobre cada sessão de interação à distância.

\subsection{Procedimentos}

Os procedimentos que foram adotados para a coleta dos dados ocorrerão em 3 etapas, a saber:

Etapa 1 - Estabelecimento de uma agenda com os participantes

Depois de identificados os participantes, uma agenda foi estabelecida para a realização das tarefas propostas neste trabalho. Em seguida, Os participantes assinaram o formulário de consentimento e preencheram o formulário de identificação.
Etapa 2 - Interação dos participantes no LMS Amadeus

Esta fase objetivou observar o nível de aceitação e compreensão dos alunos quanto ao ambiente, e registrar a percepção social destes em relação ao LMS Amadeus durante o curso "[IFPE- Belo Jardim] Capacitação Amadeus".

Etapa 3 - Grupo Focal (Focus Group) com os participantes

A utilização da técnica grupo focal, neste trabalho, objetivou proporcionar um momento para os participantes expressarem e discutirem coletivamente suas opiniões quanto a sua experiência de percepção e aceitação no ambiente. O sentido era validar e consensualizar os resultados obtidos a partir dos questionários aplicados.

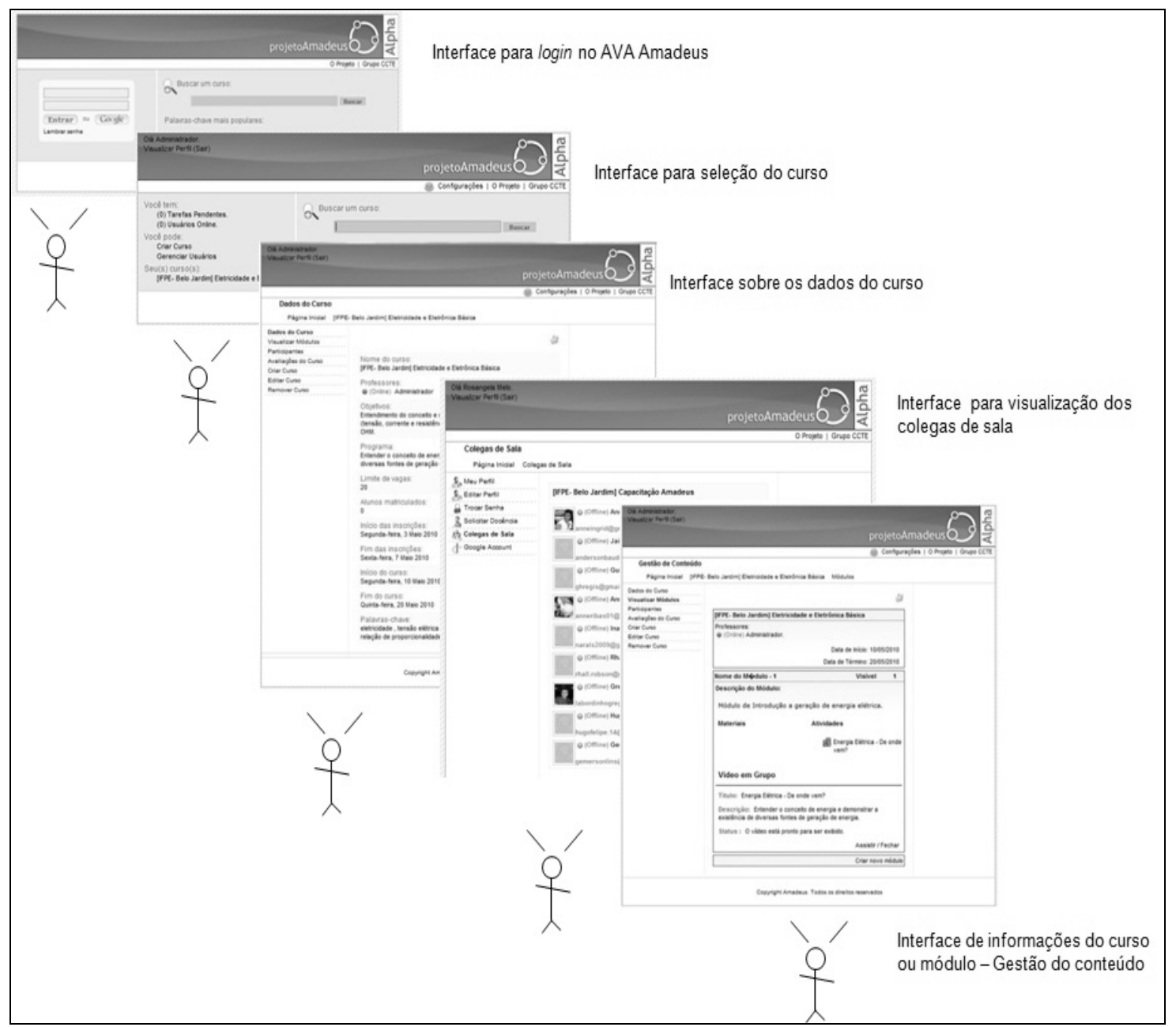

Figura 1: Contexto da pesquisa no LMS Amadeus. 


\subsection{Técnicas}

As técnicas utilizadas para atingir os objetivos propostos foram:

\section{Observação}

Segundo [8], este tipo de coleta é complexo para os seres humanos explicarem ou descreverem precisamente como os usuários realizam uma tarefa. Por outro lado, a observação natural oferece percepções que outras técnicas não podem oferecer. Sendo assim, durante todas as fases do procedimento, foi utilizada a técnica de observação, através de anotações manuais e gravações em vídeo, com o objetivo de registrar manualmente as atividades dos alunos, permeando assim, de forma complementar, todos os momentos de interação com o LMS Amadeus.

\section{Questionário}

De acordo com [12], a técnica de questionários: “[...] constituem uma técnica bem estabelecida de coleta de dados demográficos e de opiniões de usuários. São semelhantes a entrevistas e podem conter perguntas abertas ou fechadas".

O questionário foi aplicado após a conclusão da Etapa 2 - Interação dos participantes no LMS Amadeus e teve como objetivo registrar a percepção dos alunos em relação ao ambiente de aprendizagem e entre os pares. $\mathrm{O}$ questionário tratou de questões relacionadas a: identificar as dificuldades de encontrar os pares no ambiente, distinguir os perfis, identificar funções, informações e recursos considerados importantes a serem incluídos no LMS Amadeus, de modo a tornar a participação de todos mais efetiva no ambiente.

Este trabalho é resultante do esforço em contribuir para o aperfeiçoamento dos elementos de percepção no LMS Amadeus, de forma possibilitar ao usuário a verdadeira sensação de colaboração e comunidade, fazendo sentir-se inserido no grupo.

Na próxima seção são apresentados e discutidos os resultados baseados nos procedimentos metodológicos utilizados nesta pesquisa.

\section{Resultados}

De acordo com o procedimento metodológico aplicado nesta pesquisa e considerando os objetivos definidos, a seguir são apresentados os resultados que se restringem as etapas de login até a obtenção das informações do curso ou módulo no LMS Amadeus.

Os alunos em suas respostas disseram que a exploração no ambiente foi fácil, flexível, estimulante e satisfatória. De uma maneira geral, este fornece elementos que auxiliam, facilitam e estimulam o aluno a utilizá-lo.

As respostas dos questionários permitiram mapear as dificuldades dos alunos em perceber os seus pares. Foi possível avaliar o processo de identificação das funções, informações e recursos considerados importantes a serem incluídos de forma a favorecer a manutenção das relações sociais no LMS Amadeus.

As dificuldades foram identificadas durante a observação e ratificadas através do histórico de captura de tela. Há sinais da existência de dificuldades no entendimento para navegação no ambiente. Alguns alunos permaneceram mais tempo que outros para perceberem as informações contidas nas interfaces e assim, prosseguir com a exploração. Esta observação foi confirmada quando da realização do grupo focal.

A probabilidade de manter contato com os seus colegas, mesmo que estes não estejam online no LMS Amadeus foi apontada pelos alunos como um fator que pode auxiliar na manutenção das relações no ambiente, visto que esta seria uma forma adicional de comunicação. Assim, os alunos foram questionados a respeito das possíveis formas capazes de facilitar e complementar a sua comunicação, com vistas a desenvolver suas atividades.

Dentre as possibilidades mais selecionadas pelos alunos destacam-se: a possibilidade de integração com redes sociais. Isto denota o quanto os alunos pesquisados têm conhecimento da existência de tais recursos e da sua potencialidade de uso.

A integração do LMS Amadeus com as redes social tais como: Orkut, Facebook, Twitter é vista pelos alunos como uma possibilidade de extensão do ambiente. Para [13]: As novas oportunidades das "redes de relacionamento social via web”, [...] são compatíveis com a participação em cursos online. Todas as características do chamado web 2.0, ou a mais recente geração de formas de usar a rede para atividades sociais, estão alinhadas com as novas maneiras de adquirir conhecimento e competências, especialmente as que incluem ênfase ao trabalho colaborativo, compartilhamento de informação e alto grau de interatividade.

Isto pode ser constatado no trabalho de [14]. O autor propõe a agregação de uma ferramenta social no LMS Amadeus - através do Twitter - que permita atualizações rápidas e curtas sendo possível viabilizar a comunicação entre professores e aprendizes de forma rápida e eficaz. $\mathrm{O}$ autor complementa: [...] aplicações pedagógicas do twitter podem ser relacionadas diretamente ao professor como publicação de materiais, localizar idéias e frases originais, retorno aos alunos como forma de refinar seus pensamentos e competências e construção de uma comunidade ativa, onde cada participante pode contribuir com o grupo utilizando os diversos meios de interação 
possíveis, provando ser uma ferramenta eficaz no sentido da interação e colaboração entre os participantes do processo, em contextos educativos.

As redes sociais (Orkut, Facebook e Twitter) aglomeram muitas comunidades virtuais e que, a partir destas muitos saberes são produzidos. Assim, a sua adoção permitiria aos participantes a comunicação com os seus pares através de uma atividade simples, divertida e colaborativa.

Os alunos ressaltaram que este tipo de integração é importante no sentido de estender o processo de comunicação entre os pares para o desenvolvimento e registro das atividades propostas pelo professor. Entretanto, expu- seram que tal funcionalidade deve permitir o registro de informações no mural de recados do aluno, desde que este a habilite em seu perfil.

O estabelecimento de comunicação entre os alunos através do ambiente - seja através redes sociais, dispositivos móveis e utilização de mensagens gravadas de áudio e vídeo - mostram-se em consonância com o relatório de [15] que evidencia as tendências para as práticas educacionais do futuro, e que são ratificadas por [16].

Inicialmente são apresentadas as Figuras 2 e 3 que descrevem as interfaces: login no LMS Amadeus e Seleção do Curso, seguidas pelo Quadro que 2 que relaciona as necessidades identificadas nessas interfaces.

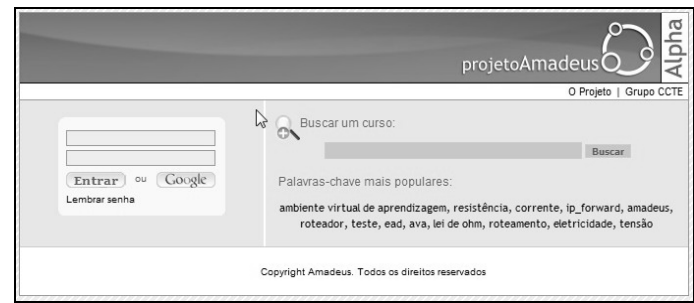

Figura 2: Login no LMS Amadeus.

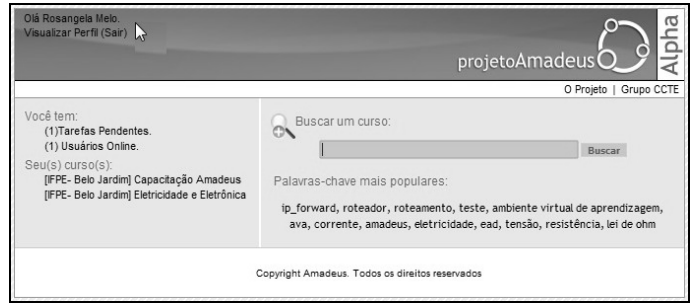

Figura 3: Seleção do curso.

\begin{tabular}{|c|c|c|}
\hline & Descrição das Necessidades & Interface \\
\hline 1 & $\begin{array}{l}\text { A mensagem "Buscar um curso" não é clara. O mecanismo de busca da } \\
\text { interface de entrada deve fornecer clareza e objetividade para o usuá- } \\
\text { rio. }\end{array}$ & Figuras 2 e 3 \\
\hline 2 & $\begin{array}{l}\text { Possibilidade de realçar as palavras-chave de forma a dar entender que } \\
\text { são categorias de cursos disponíveis no ambiente, pois da forma como } \\
\text { se encontra, fornece a impressão de serem apenas informações textuais } \\
\text { e não categorias. }\end{array}$ & Figuras 2 e 3 \\
\hline 3 & $\begin{array}{l}\text { Possibilidade de interação com os usuários online, independente do } \\
\text { curso que estejam vinculados. }\end{array}$ & Figura 3 \\
\hline 4 & $\begin{array}{l}\text { Possibilidade de indicações diretas sobre a oferta e andamento de } \\
\text { cursos ou módulos, após o login do aluno no LMS Amadeus. }\end{array}$ & Figura 3 \\
\hline 5 & Possibilidade da indicação de presença dos pares durante a navegação. & Figura 3 \\
\hline
\end{tabular}

Quadro 2: Necessidades identificadas nas interfaces login e seleção do curso.

As Figuras 4, 5 e 6 apresentam a sequência de telas que identificam as interfaces: Usuários online, Perfil do Aluno e Perfil do Usuário no Ambiente, respectivamente, seguidas pelo Quadro 3 que lista as necessidades identificadas nessas interfaces.
As interfaces supracitadas são acessadas partir da interface representada na Figura 3, após a seleção do item "Usuários Online" no LMS Amadeus 


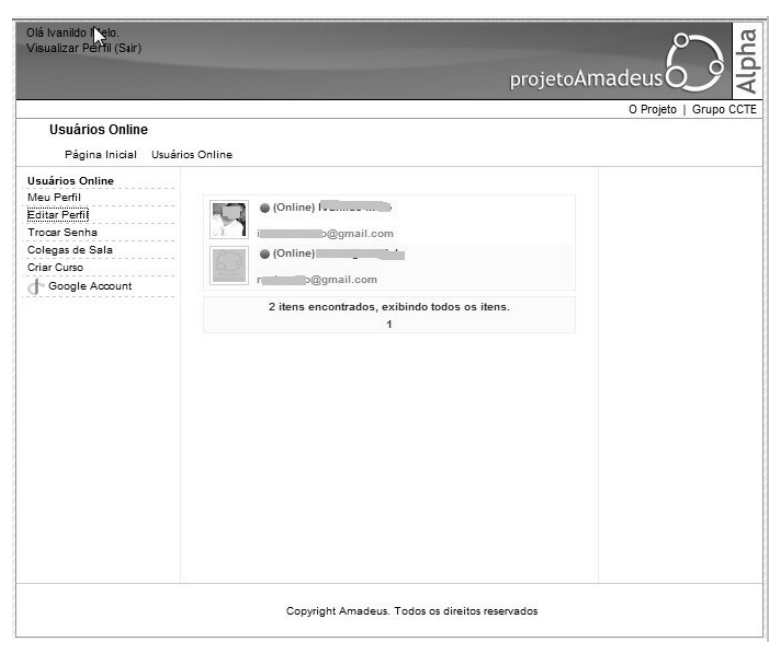

Figura 4: Usuários online.

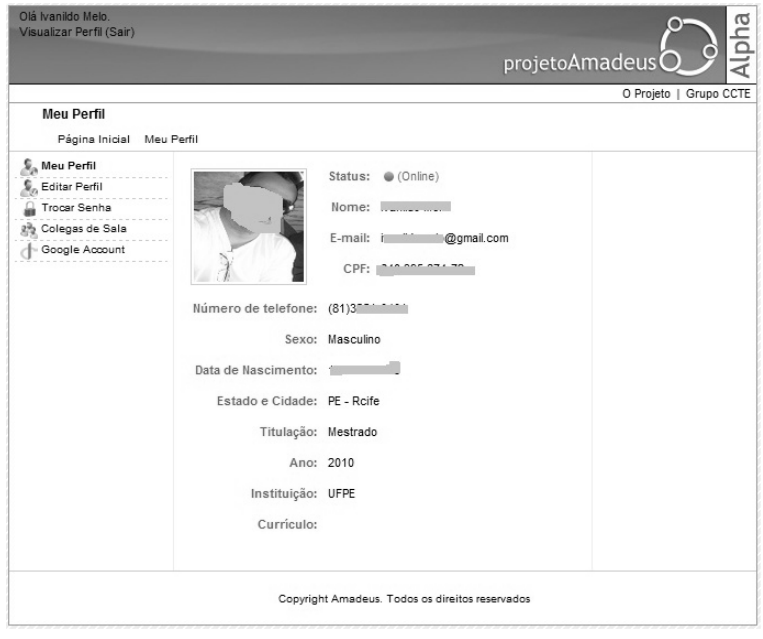

Figura 5: Perfil do aluno.

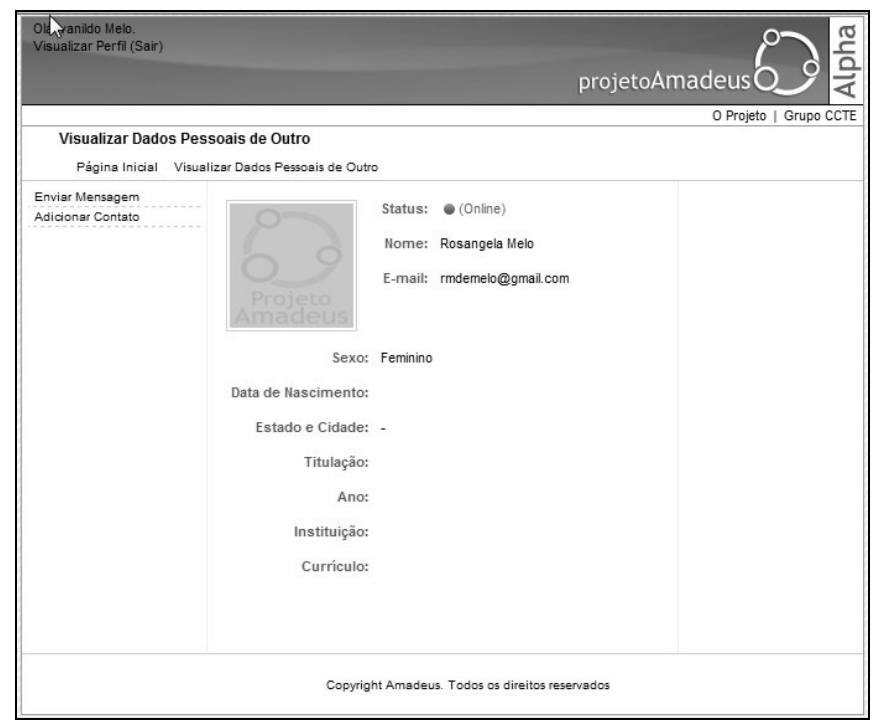

Figura 6: Perfil de outro usuário no ambiente.

\begin{tabular}{|c|l|c|}
\cline { 2 - 4 } \multicolumn{1}{c|}{} & \multicolumn{1}{|c|}{ Descrição das Necessidades } & Interface \\
\hline 1 & Indicação da presença dos pares durante a navegação. & Figuras 4 e 6 \\
\hline 2 & Serviço de mensagem no ambiente & Figuras 4 e 6 \\
\hline 3 & Integração à redes sociais Orkut, Facebook, Twitter. & Figuras 4 e 6 \\
\hline 4 & Envio de mensagens SMS - Short Mensage Service. & Figuras 4 e 6 \\
\hline
\end{tabular}

Quadro 3: Necessidades identificadas nas interfaces usuários online, perfil do aluno e perfil de outro usuário no ambiente.

As Figuras 7 e 8 apresentam a sequência de telas que identificam as interfaces: Dados do Curso e Gestão do Conteúdo, seguidas pelo Quadro 4 que enumera as neces- sidades identificadas nessas interfaces. Estas são geradas partir da Figura 3, após a seleção do curso no qual o aluno está matriculado no LMS Amadeus. 


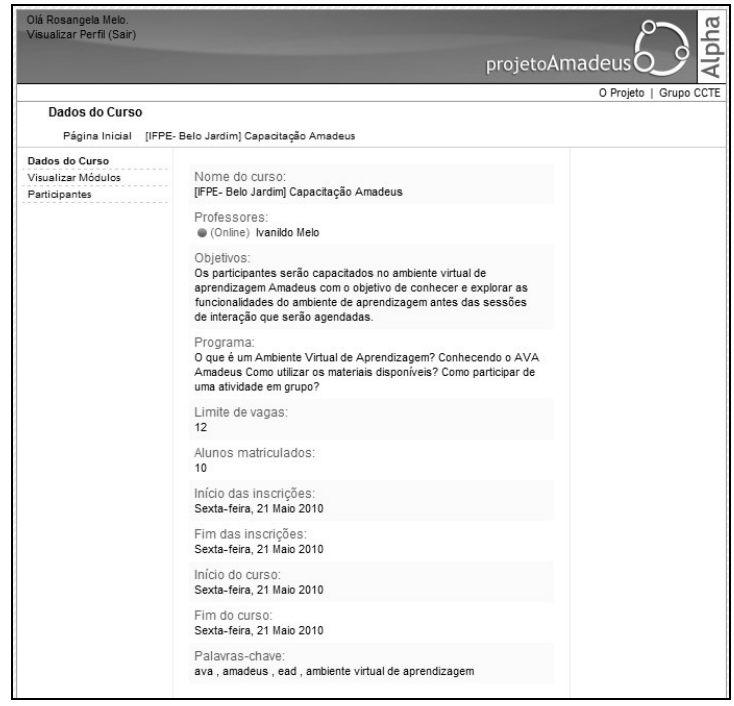

Figura 7: Dados do curso

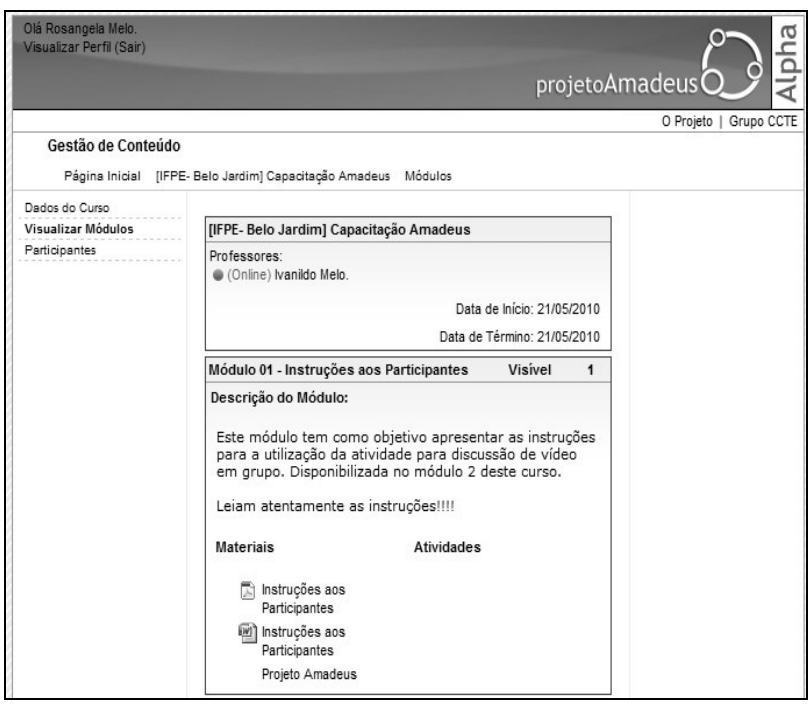

Figura 8: Gestão do conteúdo.

\begin{tabular}{|l|l|l|}
\cline { 2 - 3 } \multicolumn{1}{c|}{ Descrição das Necessidades } & \multicolumn{1}{|c|}{ Interface } \\
\hline 1 & $\begin{array}{l}\text { Interação com os seus pares e com o professor, a partir da indica- } \\
\text { ça do Status. }\end{array}$ & Figura 7 e 8 \\
\hline 2 & $\begin{array}{l}\text { Indicação de presença dos participantes durante a navegação. } \\
\text { Figura } 7 \text { e } 8\end{array}$ \\
\hline
\end{tabular}

Quadro 4: Necessidades Identificadas nas Interfaces Dados do Curso e Gestão do Conteúdo.

A Figura 9 apresenta a tela que identifica os participantes de um curso no LMS Amadeus, seguida pelo Quadro 5 que trata das necessidades identificadas nessa inter- face. $\mathrm{O}$ acesso a esta interface é realizado a partir das interfaces representadas pelas Figuras 7 e 8 através da opção "Participantes" disponível no menu.

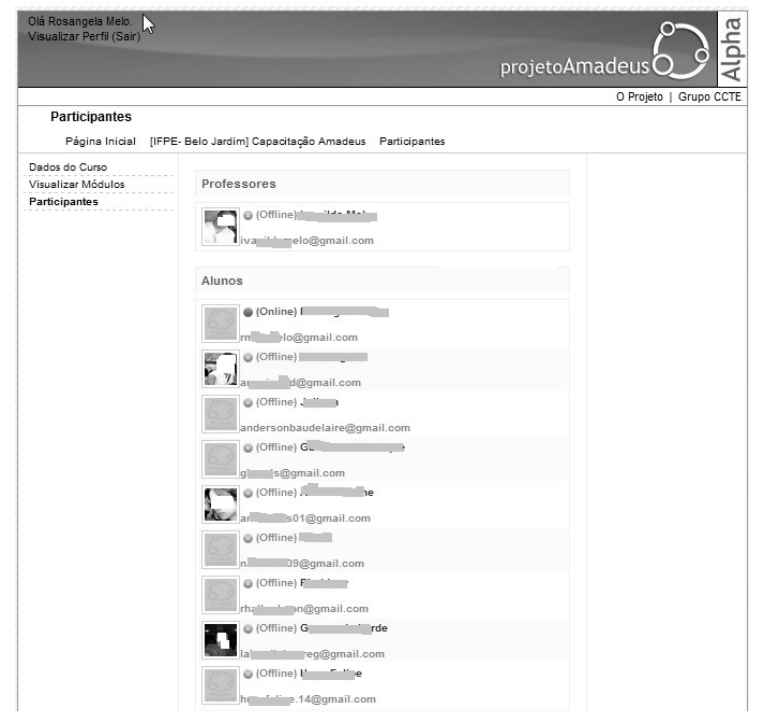

Figura 9: Participantes de um curso. 


\begin{tabular}{|c|l|c|}
\cline { 2 - 3 } \multicolumn{1}{c|}{ Descrição das Necessidades } & Interface \\
\hline 1 & $\begin{array}{l}\text { Interação com os seus pares e com o professor a partir da indica- } \\
\text { ça do Status. }\end{array}$ & Figura 9 \\
\hline 2 & Integração redes sociais Orkut, Facebook, Twitter. & \\
\hline 3 & Envio de mensagens através de SMS. & \\
\hline 4 & Indicação de presença dos participantes durante a navegação. \\
\hline
\end{tabular}

Quadro 5: Necessidades identificadas na interface participante.

A utilização da técnica na Etapa 3 - Grupo Focal (Focus Group) com os participantes - permitiu a validação e um aparente consenso dos resultados obtidos a partir dos questionários aplicados e são apresentadas no Quadro 6.

\begin{tabular}{|c|c|c|}
\hline & Descrição das Necessidades & Interface \\
\hline 1 & $\begin{array}{l}\text { Possibilidade de serviço de mensagens. O serviço de mensagem } \\
\text { disponível no LMS Amadeus trata-se do envio de email, vide Fi- } \\
\text { gura 8. A versão utilizada na pesquisa não permitiu a sua uti- } \\
\text { lização. Os alunos sugerem que este serviço seja mantido no } \\
\text { ambiente. }\end{array}$ & $\begin{array}{l}\text { Figuras } 4,6,7,8 \\
\text { e } 9\end{array}$ \\
\hline 2 & $\begin{array}{l}\text { Envio de mensagens para o mural de recados de redes sociais } \\
\text { Orkut, Facebook, Twitter. Esta funcionalidade seria ativada } \\
\text { opcionalmente pelo aluno quando este a desejasse. }\end{array}$ & $\begin{array}{l}\text { Figuras } 4,6,7,8 \\
\text { e } 9\end{array}$ \\
\hline 3 & $\begin{array}{l}\text { Envio de mensagens pré-formatadas através de SMS. Esta funcio- } \\
\text { nalidade seria ativada, opcionalmente, pelo aluno quando este a } \\
\text { desejasse. }\end{array}$ & $\begin{array}{l}\text { Figuras } 4,6,7,8 \\
\text { e } 9\end{array}$ \\
\hline 4 & $\begin{array}{l}\text { Interação do aluno com os seus pares e deste com o professor, a } \\
\text { partir da indicação do status deve ser limitada a duas sessões. }\end{array}$ & $\begin{array}{l}\text { Figuras } 4,6,7,8 \\
\text { e } 9\end{array}$ \\
\hline 5 & $\begin{array}{l}\text { Realce nas palavras-chave - tamanho de fontes e cores diferen- } \\
\text { tes. }\end{array}$ & Figuras 2 e 3 \\
\hline
\end{tabular}

Quadro 6: Necessidades consensualizadas no grupo focal.

A última etapa do grupo focal foi finalizada com o convite aos alunos para que estes sugerissem protótipos com vistas a aperfeiçoar as interfaces do LMS Amadeus nas quais eles interagiram. Isto permitiu que os alunos fornecessem um feedback informátivo sobre suas compreensões em relação ao ambiente, e ainda participar ativamente das discussões durante a avaliação dos protótipos.

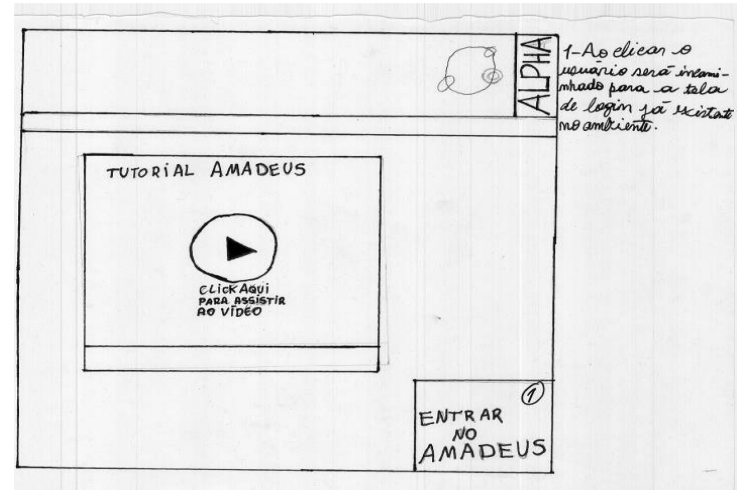

Figura 10 - Protótipo em papel sugerido pelos participantes.
Para tanto, foi fornecido cartolina, pinceis de cores diversas. Os alunos agruparam-se e sugeriram protótipos e modificações. Alguns destes estão representados pelas Figuras 10 e 11 . Além disto, esta etapa foi finalizada com a identificação algumas necessidades que emergiram quando da realização do grupo focal e que estão descritas no Quadro 7 .

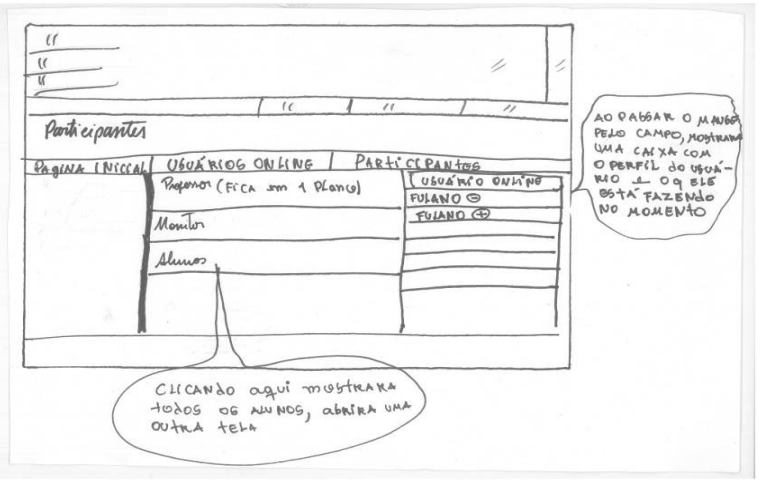

Figura 11- Protótipo em papel sugerido pelos participantes. 


\begin{tabular}{|c|c|c|}
\hline & Descrição das Necessidades & Interface \\
\hline 1 & $\begin{array}{l}\text { Alteração da cor da indicação status de presença a partir de um } \\
\text { tempo pré-determinado. }\end{array}$ & $\begin{array}{l}\text { Figuras } 3,4,6,8 \\
\text { e } 9\end{array}$ \\
\hline 2 & $\begin{array}{l}\text { Separação dos contextos da interface de forma a facilitar a } \\
\text { visualização, evitando a sensação de texto único. }\end{array}$ & $\begin{array}{l}\text { Figuras } 4,6,7,8 \\
\text { e } 9\end{array}$ \\
\hline 3 & $\begin{array}{l}\text { Disponibilização de histórico de atividades e de acesso no am- } \\
\text { biente. Esta necessidade seria opcionalmente ativada no ambien- } \\
\text { te pelo aluno. }\end{array}$ & $\begin{array}{l}\text { Figuras } 3,4,6, \\
7,8 \text { e } 9\end{array}$ \\
\hline 4 & $\begin{array}{l}\text { Dispor ao professor relatórios do histórico de acesso e de ati- } \\
\text { vidades dos alunos para facilitar o acompanhamento da turma. }\end{array}$ & \\
\hline
\end{tabular}

Quadro 7: Necessidades surgidas no grupo focal.

\section{Requisitos Estabelecidos}

Baseado na análise qualitativa dos dados, são apresentados no Quadro 8, os requisitos que foram estabelecidos a partir da identificação das dificuldades e das necessidades expressadas pelos participantes durante a realização dos procedimentos.
Foram estabelecidos 12 requisitos, relacionados às interfaces de navegação, desde a interface de login até a interface que contém informações do curso ou módulo.

O Quadro 8 a seguir descreve a correspondência entre os requisitos e os protótipos que foram concebidos.

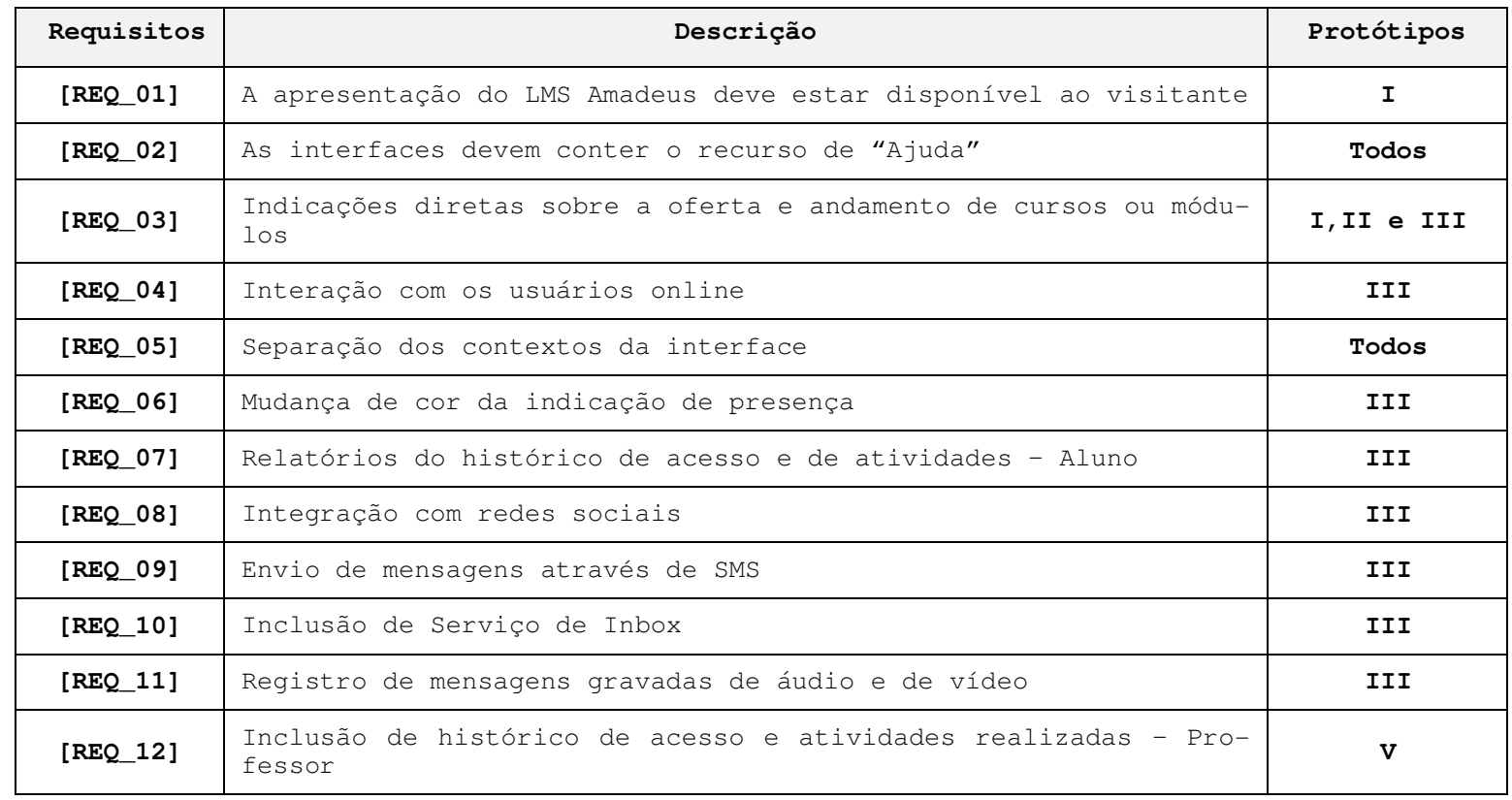

Quadro 8: Requisitos estabelecidos a partir das necessidades identificadas.

\section{Protótipos Sugeridos}

Esta etapa consistiu na construção de protótipos de telas, que apontaram sugestões a serem implementadas de acordo com os aspectos de percepção e de colaboração identificados nos requisitos, bem como nas sugestões prototipadas pelos participantes, ao final do grupo focal.

Em um momento seguinte, os protótipos foram apresentados aos alunos, em papel impresso, e a avaliação de cada interface permitiu descrever problemas de comuni- cabilidade com os mesmos. A avaliação também contribuiu para elucidar dúvidas quanto aos protótipos sugeridos no grupo focal com os participantes.

O processo avaliativo fez com que os alunos interagissem com os protótipos e discutissem coletivamente buscando a validação das funcionalidades propostas, como também, permitiu a discussão e a busca para resolução refinada dos problemas encontrados. As figuras 12,13 , 14,15 e 16 representam alguns dos protótipos sugeridos, e que foram avaliados pelos participantes. 

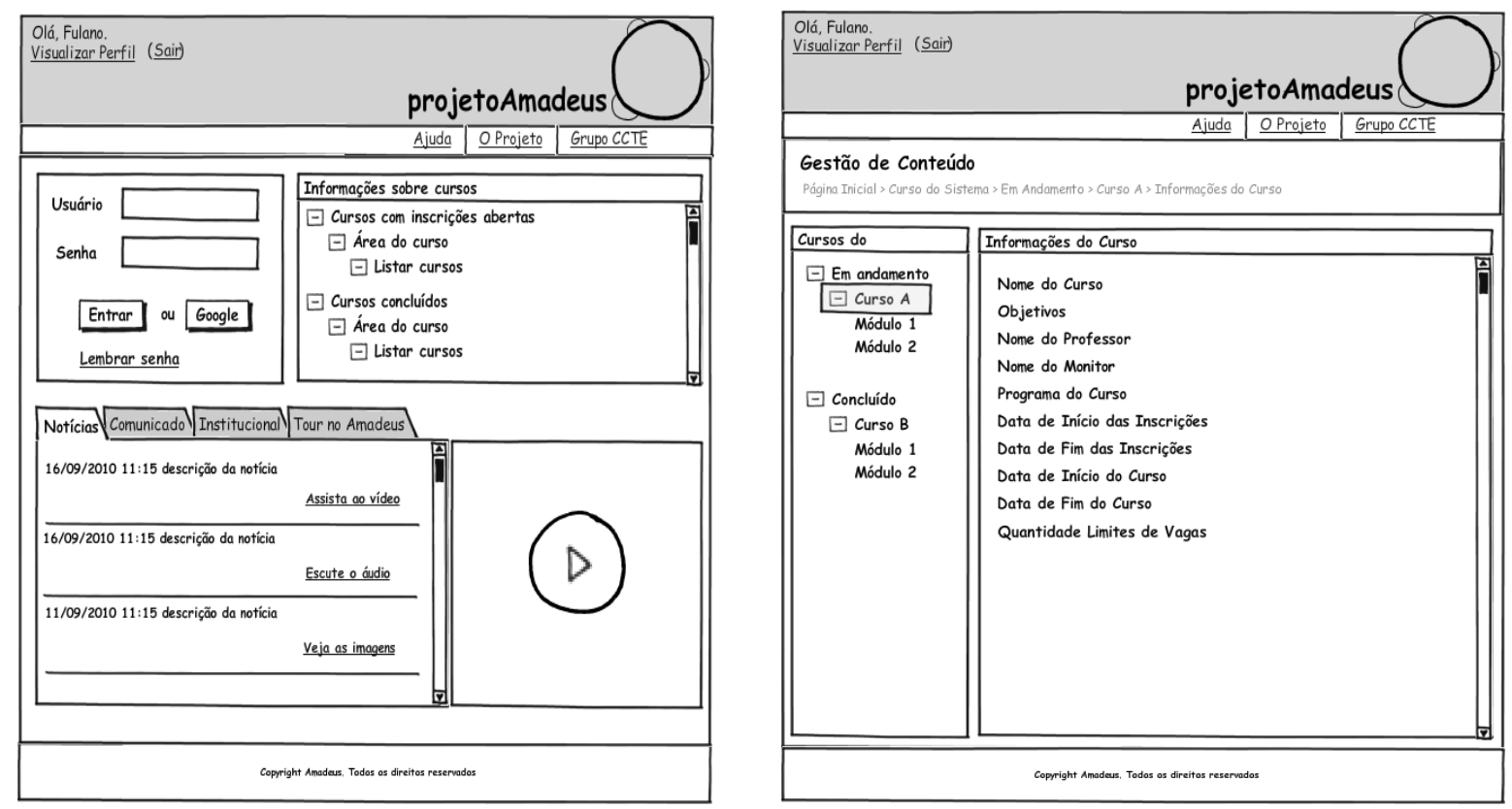

Figura 12: Protótipo I - Interface de entrada no LMS Amadeus. Figura 13: Protótipo II - Informações sobre curso/módulo.

\begin{tabular}{|c|c|c|c|}
\hline \multicolumn{4}{|l|}{$\begin{array}{l}\text { Olá, Fulano. } \\
\text { Visualizar Perfil (Sair) }\end{array}$} \\
\hline \multicolumn{2}{|r|}{ Ajuda } & OProjeto & Grupo CCTE \\
\hline \multicolumn{4}{|c|}{$\begin{array}{l}\text { Gestão de Conteúdo } \\
\text { Página Inicial > Curso do Sistema }>\text { Em Andanentio }>\text { Curso A> Módulo } 1\end{array}$} \\
\hline Cursos do & \multicolumn{3}{|l|}{\begin{tabular}{|l} 
Módulo 1 \\
\end{tabular}} \\
\hline \multirow[t]{3}{*}{$\begin{array}{l}\square \text { Em andamento } \\
\square \text { Curso A } \\
\text { Módulo 1 } \\
\text { Módulo 2 } \\
\square \text { Concluído } \\
\square \text { Curso B } \\
\text { Módulo 1 } \\
\text { Módulo 2 }\end{array}$} & \multicolumn{3}{|l|}{$\begin{array}{l}\text { Nome do Módulo } \\
\text { Descrição do Módulo } \\
\text { Data de Início do Módulo } \\
\text { Data de Fim do Módulo } \\
\text { Materiais } \\
\text { Atividades } \\
\begin{array}{|l}\text { Histórico de Acesso } \\
\text { Nome / Data / Hora / Descrição }\end{array}\end{array}$} \\
\hline & Usuários & $0 \mathrm{fB}$ & @ 9 \\
\hline & 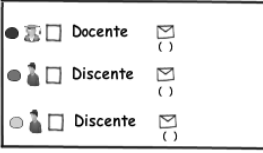 & $\begin{array}{lll} & \mathbf{f} \\
0 & \mathrm{~B} \\
0 & \mathbf{f} \\
\end{array}$ & $\begin{array}{lll}9 & \square \\
& (1) & 1 \\
& & \\
& & 1 \\
0 & 9 & \\
(1) & \end{array}$ \\
\hline
\end{tabular}

Figura 13: Protótipo III - Histórico de acesso.

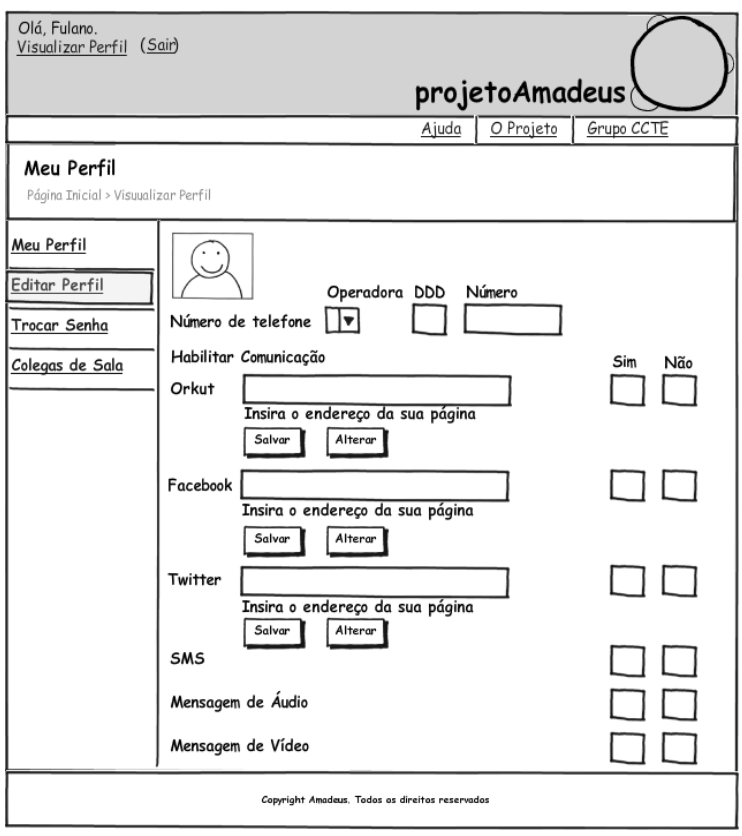

Figura 15: Protótipo IV - Configurações do perfil do participante. 


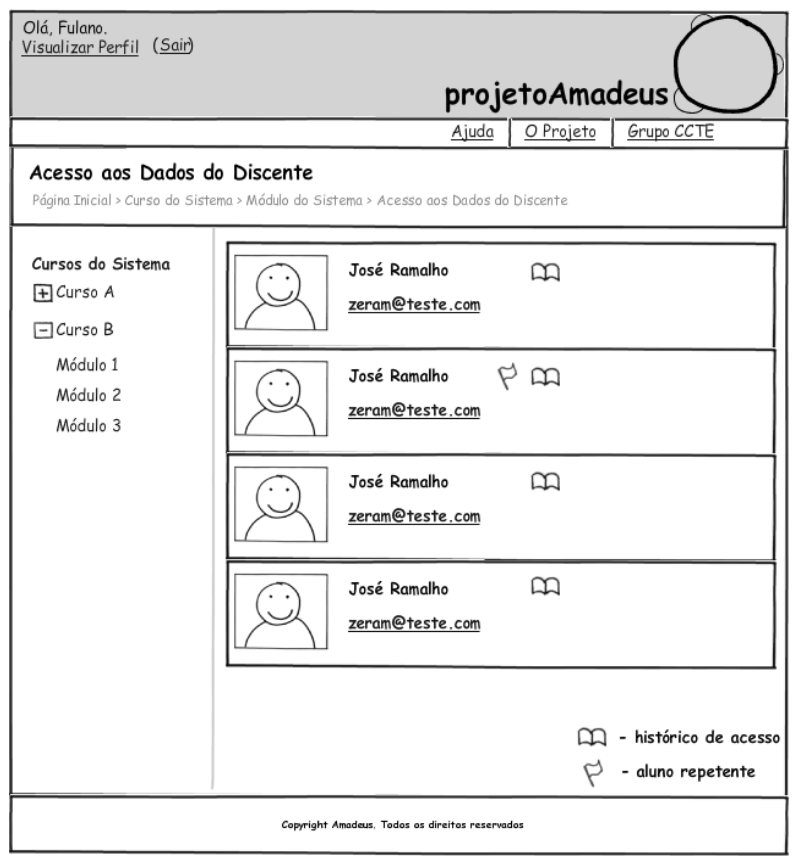

Figura 14: Protótipo V - Acesso aos dados do discente.

\section{Conclusões}

Este trabalho tem como propósito contribuir para o aperfeiçoamento dos elementos de percepção no LMS Amadeus, de forma a possibilitar ao usuário a verdadeira sensação de colaboração e comunidade, fazendo-o sentirse inserido no grupo.

O contexto do LMS Amadeus avaliado, neste trabalho, sinalizou que os elementos de percepção social existentes limitam-se a indicação da quantidade dos usuários no ambiente e do seu status. Isto torna a comunicação e o engajamento dos alunos difícil, para não dizer inexistente.

As necessidades identificadas no LMS Amadeus apontaram que os atuais mecanismos de percepção social precisam ser complementados, da mesma forma que outros necessitam ser integrados ao ambiente. Há indícios que essas melhorias beneficiarão a comunicação, e consequentemente a participação dos alunos, além de promover a redução da sensação de solidão comum aos participantes em ambientes virtuais de aprendizagem.

Apesar da maioria dos alunos afirmarem que a exploração das interfaces apresenta níveis satisfatórios de utilização, e que se sentem estimulados a utilizá-lo, foram identificadas necessidades para sua melhoria.

No decorrer do grupo focal foram sugeridas mudanças no design de algumas interfaces, tendo em vista que estas não deixam claro o limite textual, o que confunde o aluno quando da sua utilização, a exemplo da Figura 7. Apesar do foco desta pesquisa ser as necessidades dos alunos, estes se preocuparam com a visão do professor, e sugeriram a existência de relatórios que evidenciem o histórico de acesso e de atividades dos alunos para o professor. Esta sugestão visa facilitar o acompanhamento do aluno e da turma.

Os resultados deste trabalho foram reportados a comunidade do Projeto Amadeus através do Portal do Software Público Brasileiro. Os próximos passos para a continuidade da pesquisa estarão centrados na concepção de protótipos para a apresentação e avaliação pela comunidade do Projeto Amadeus.

É importante ressaltar que o processo de avaliação será contínuo, de forma que as necessidades identificadas possam ser futuramente incorporadas. E, a partir destas, novas avaliações possam ser realizadas, com vistas ao aprimoramento do LMS Amadeus.

Os resultados obtidos não estão limitados apenas ao LMS Amadeus. As necessidades identificadas nesta pesquisa podem vir a ser constatadas em outros Learning Management Systems, não esquecendo, sobretudo, que este processo deve respeitar as capacidades, necessidades e preferências dos seus usuários. 


\section{Referências}

[1] Carvalho, R. S. Sistemas de gestão da Aprendizagem e Sistemas de Gestão Acadêmica: Avaliados pela Ótica do Docente. 2010. Dissertação de Mestrado. Universidade Federal de Pernambuco - Centro de Informática - Pós-Graduação em Ciência da Computação.

[2] Rittenbruch, M., McEwan G. (2009) An Historical Reflection of Awareness in Collaboration Chapter 1 - Awareness Systems - Advances in Theory, Methodology, and Design - HumanComputer Interaction Series - Markopoulos, P., Ruyter, B. de, Mackay, W. (Editors) - Springer.

[3] Gerosa, M.A., Fuks, H. \& Lucena, C.J.P. (2003) Suporte à Percepção em Ambientes Digitais de Aprendizagem, Revista Brasileira de Informática na Educação, Vol. 11, No. 2, Novembro 2003, ISSN 1414-5685

[4] Prasolova-Forland E. (2002) Supporting awareness in education: overview and mechanisms. International Conference on Engineering Education August 18-21, 2002, Manchester, U.K.

[5] Pilati, R. Percepção Social - Laboratório de Psicologia Ambiental - Instituto de Psicologia Universidade de Brasília. 2007.

[6] Idrus Z., Abidin S., Hashim R., OMAR N. Social awareness: the power of digital elements in collaborative environment. WSEAS Transactions on Computers 9 (6): 644-653, 2010.

[7] Markopoulos, P., Ruyter, B. de, Mackay, W. (2009) Awareness Systems - Advances in Theory, Methodology, and Design - Human - Computer Interaction Series - Springer.

[8] Melo Filho, I. J. Percepção Social em Interfaces Distribuídas para o Aprendizado Online. (2010). Dissertação de Mestrado. Universidade Federal de Pernambuco - Centro de Informática - PósGraduação em Ciência da Computação.

[9] Gomes, A.S; Monteiro, B.S.; Carvalho, R.S; Melo Filho, I.J.; Rolim, A.L.S.; Oliveira, G.R.S. AMADEUS - Novas Formas de Interação para Educação a Distância. Revista Espírito Livre Edição 008 - Páginas 73 - 78. Novembro/2009.

[10] Wazlawick, R. S. Metodologia de pesquisa para ciência da computação. Editora Elsevier. Rio de Janeiro. 2008.
[11] Gil, A. C. Como elaborar projetos de pesquisa. Editora Atlas. 4a Edição. São Paulo. 2009.

[12] Preece, J.; Rogers, Y.; Sharp, H. - Design da Interação - Além da Interação Homem - Computador. Porto Alegre/RS. Editora Bookman, 2005.

[13] Litto, F. M. - Aprendizagem a distância. Editora Imprensa Oficial. São Paulo. 2010.

[14] Teixeira, E. O. - Estilos de Interação em EAD: O Microblogging Twitter. Dissertação (especialização) - Faculdade de Ciências Aplicadas e Sociais de Petrolina. CAPG. Especialização em Engenharia de Software. Petrolina. 2009.

[15] New Media Consortium and Educause Learning Initiative, em The Horizon Report. 2007. Disponível em:< http://www.nmc.org/horizon/2007/ technologies-watch>. Acesso em: 10 out. 2010.

Tori, R. - Educação sem distâncias - Tecnologi-

[16] as interativas na redução de distâncias em ensino aprendizagem. Escola do Futuro - Universidade de São Paulo. Editora SENAC. São Paulo. 2010. 